\title{
Application of BP Neutral Network and Genetic Algorithm
}

\author{
Liu Jie $^{1}$, Yan Huifeng ${ }^{2, *}$ and Pan Yingjun ${ }^{2}$ \\ ${ }^{I}$ School Of Civil Engineering \& Architecture, Chongqing Jiaotong Univ., Chongqing, 400074, P.R. China; ${ }^{2}$ The key lab \\ of OptoElectronics Technology and System, Ministry of Education, ChongQing University, Chongqing, 400044, P.R. \\ China
}

\begin{abstract}
Ro-Ro transportation refers to a way of maritime transportation that uses Ro-Ro to bring goods and shipment together. Developments of Ro-Ro transport at home and abroad are outlined, and development process of China's Ro-Ro transport is reviewed. The development and status of Ro-Ro transport of Yangtze River are analyzed in detail. Based on survey research, due to the change of road structure of Yangtze River region, the survival and development of Yangtze River Ro-Ro transport are faced with serious threats. It is imperative to forecast the future freight volume of Yangtze River Ro-Ro transport with assured accuracy, and generate strategies to ensure the healthy development of Yangtze River Ro-Ro transport. This paper adopts the GABP (Genetic-BP Neural Network) model to forecast the freight volume of Yangtze River Ro-Ro transport. Comparative data is used to ensure the accuracy of the model, which solves the imbalances of traffic and capacity.
\end{abstract}

Keywords: Ro-Ro Transportation Market, Traffic, Genetic BP neural network.

\section{INTRODUCTION}

Ro-Ro transport is the use of the truck, semi-trailer or a truck carrying cargo and Ro-Ro transport by truck, making it a multimodal approach to reach the destination [1]. The rapid rise in recent years in the Yangtze River cargo ship on the Yangtze River basin, with its fast, convenient, on the wharf infrastructure or ship lifting equipment less demanding, lowcost, environmentally friendly, applicability, and other characteristics, is rapidly becoming the new Yangtze River shipping highlights for the Yangtze River shipping industry injection of fresh blood. Since 2004, the Yangtze River Ro-Ro transport market in recent years after a boom period after its internal problems because of market management system is not perfect, the specific performance such as poor infrastructure, poor market management, policy support is not enough and so on. While the external environment changes, can cause external problems, the market mainly refers to changing external problems along the road structure. Since 2008, along the parallel construction of highways and railways, changing the structure of the Yangtze River overland route may bypass Ro-Ro market share of the transport market, the development of markets such as Ro-Ro transport of threat. While developing the transport companies emerged early, unlimited expansion of capacity, while structural changes may reduce Ro-Ro road transport market share, will result in more than traffic capacity, resulting in an imbalance volume capacity, will produce a series of serious consequences, such as capacity waste of resources, vicious competition within the market, such as ab-normal development of Ro-Ro transport market. Hence the need to address the Yangtze River Ro transportation market both internal and external problems, solving internal problems to ensure the sustainable development of Ro-Ro transport market to solve external problems to ensure the survival of Ro-Ro transport market. The main problem is the volume of external forecasting problems, the next year or several years, it is particularly important cargo, with the volume of data information, you can of Ro-Ro shipping market capacity for the deployment, reducing the capacity of the waste of resources, from comprehensive to local planning market, thus guarantee the survival of the Ro-Ro transport market. Therefore, the Yangtze River Ro transportation market volume forecast is particularly important. Use of scientific methods variation mining Ro transportation market, timely and accurately predict market trends and traffic in the next few years is important for Ro-Ro cargo transportation market survival and healthy development.

Studies have shown that the combination of genetic algorithms and neural networks will be more effective for cargo forecasts strong genetic algorithm optimization ability of local and global characteristics make up the convergence rate block chat neural network. Based on genetic algorithm optimization of neural network theory proposed BP neural network model (GABP), by the algorithm to predict Ro-Ro freight market of the Yangtze River and the validity of results were analyzed [1-4].

\section{GENETIC ALGORITHM BP NEURAL NETWORK MODEL}

\subsection{Genetic Algorithm}

Genetic algorithm(GA) is to learn from biological Mechanism of natural selection and evolution groups formed adaptive global optimization algorithms. In the genetic algorithm, will be represented as individual genetic space of de- 
cision variables in the problem space by certain coding method, which is a string genotype data structure; the same objective function value will be converted into a fitness value, which is used to evaluate the merits of the individual and as a basis for genetic manipulation. Genetic operations include three operators: selection, crossover and mutation.

Select the operator to implement the principle of survival of the fittest, that is part of the current population of individuals with a certain probability of selection into a new group, the individual probability of being selected values associated with fitness, the better the individual fitness value is selected the probability is greater. Since the selection operator did not produce a new individual, so the fitness of the population will not be the best value of individual choice and improved operations.

Crossover is randomly paired individuals, and then two pairs of individuals in some way interchangeable part of the gene, resulting in new outstanding individuals.

\subsection{BP Neural Network Model [2]}

Research on BP [2] neural network is used. According to projections need to set 4 in the input layer neurons, Each input neuron represents a predictive factor.

As shown in Fig. (1), the selection of numbers of neurons in the hidden layer affects the outcome of accuracy, training time and fault tolerance. Generally, the more hidden layer neurons, the more accurate the results are, but too many neurons in the hidden layer will increase the training time while hidden layer neurons will cause an increase in network fault tolerance decreases. studies show that for the n-layer neural network, the hidden layer neurons number is at least, where $\mathrm{n}$ is the number of neurons in the input layer after the above analysis, neural network hidden layer neurons number, in order to predict the best results, the hidden layer neurons is set to 7 in the output layer, output layer nodes 1 .

According to the formula1 proposed by Homik can be determined as well as the number of nodes in the hidden layer.

$$
n=[\sqrt{2 n+m}, 2 n+m]
$$

Equation (1) for the input layer nodes, $\mathrm{m}$ is the output layer nodes.
According to the formula (1), the input and output in Fig. (1) layer nodes substitution calculated by the scope of the hidden layer nodes. 3-11 can be assigned to each $n$, and simulated by computer, can get the best numerical results for the optimal value. MATLAB2012b is used on a computer simulation experiment, the optimal value of 7 . Therefore, the network topology can determine the number of neurons in the hidden layer 7, Fig. (1) of the BP network is 4-7-1.

\subsection{Genetic Algorithm BP Neural Network Model ${ }^{[2]}$}

BP neural network algorithm is actually a gradient descent algorithm. In the adaptive learning process BP neural network algorithm converges slowly; the existence of local minimization problem; robustness bad, network stability. These defects can lead to the calculation time is longer, but the accuracy and applicability will be affected, so it is necessary to improve this single BP neural network model, the method used in this paper is the use of genetic algorithm optimization BP neural network model. The key lies in the optimization process using genetic algorithm optimization neural network weights, the first randomized to receive initial weights and the optimal solution through iteration, focusing on the right to get into the BP neural network model generation, and to overcome the slow convergence and local minimal problems in a relatively short period of time to get the global optimal solution, so that the optimization model to better and more accurately predict the function output. Step genetic algorithm optimization BP neural network is mainly population initialization, fitness function, select Options, crossover and mutation, the steps shown in Fig. (2).

\subsection{Flow of Genetic Algorithm BP Neural Network [4] Step1: Population Initialization;}

Initialize the $R=\left[r_{1}, r_{2} \cdots, r_{n}\right]$ initial population containing $\mathrm{n}$ chromosomes and its coding, coding method for realcoded, each individual contains all the neural network weights and thresholds [3]. Constitute a structure, weights, threshold value determined neural networks at the network structure is already known.

\section{Step 2: Fitness Function;}

BP neural network based on the individual to get the initial weights and thresholds, after training with BP neural

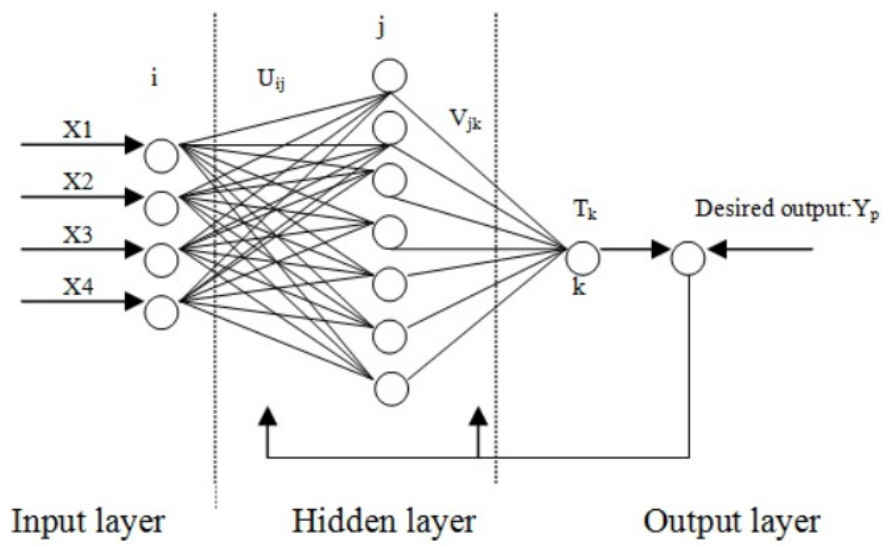

Fig. (1). BP neural network model. 


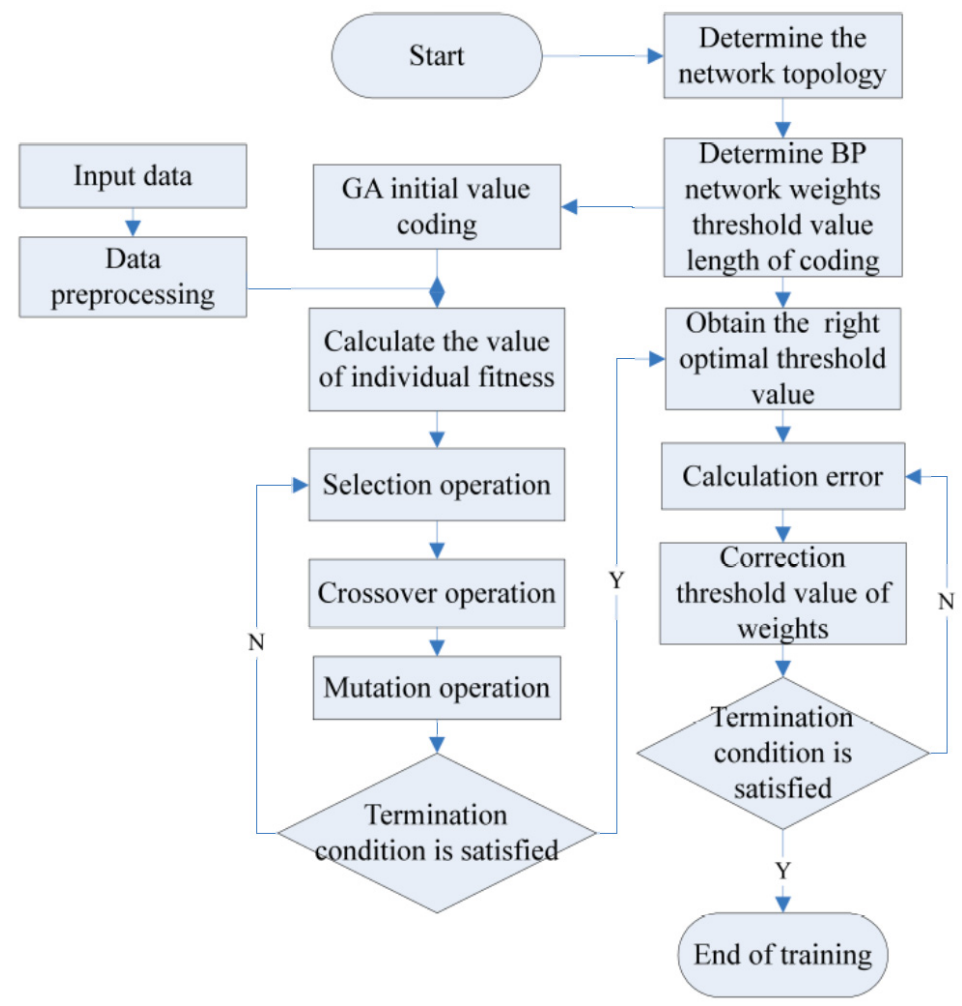

Fig. (2). GABP neural network Algorithm flowchart.

network training data to predict system output, the error between the predicted output and the desired output as the absolute value of the sum of individual fitness value $F$, the formula is:

$$
F=k\left(\sum_{i=1}^{n} a b s\left(y_{i}-o_{i}\right)\right)
$$

In equation (2), $\mathrm{n}$ is the network output nodes; $y_{i}$ is the desired output of node $\mathrm{i}$ of BP neural network; $o_{i}$ is the predicted output of the $\mathrm{i}$-th node; $\mathrm{k}$ is a coefficient.

\section{Step 3: Selection Operation;}

Selection process using roulette method, which is based on the proportion of fitness selection strategy, each individual's choice probability $y_{i}$ for $\mathrm{n}$ :

$$
\begin{aligned}
& f_{i}=\frac{k}{F_{i}} \\
& p_{i}=\frac{f_{i}}{\sum_{j=1}^{N} f_{j}}
\end{aligned}
$$

Equation (3), (4), $f_{i}$ is the fitness value of individual i, the smaller the better because of fitness, so before selecting individual fitness value of a reciprocal; $\mathrm{k}$ is a coefficient; $\mathrm{N}$ is the number of individuals in a population.

\section{Step 4: Crossover Operation;}

Due to individual use of real-coded, so crossover method using real crossover method, crossover method of chromosome and chromosome in $\mathrm{j}$ bits is as follows:

$$
\left\{\begin{array}{l}
a_{k j}=a_{k j}(1-b)+a_{l j} b \\
a_{l j}=a_{l j}(1-b)+a_{k j} b
\end{array}\right.
$$

In formula $5, \mathrm{~b}$ is a random number of $[0,1]$.

\section{Step 5: Mutation Operation;}

Select aj genes of the $\mathrm{j}$-th individual i mutate, Mutation operation as follows:

$$
\begin{aligned}
& a_{i j}=\left\{\begin{array}{cc}
a_{i j}+\left(a_{i j}-a_{\max }\right) \times f(g) & r>0.5 \\
a_{i j}+\left(a_{\min }-a_{i j}\right) \times f(g) & r \leq 0.5
\end{array}\right. \\
& f(g)=r_{2}\left(1-\frac{g}{G_{\max }}\right)^{2}(7)
\end{aligned}
$$

In equation (6) and (7), $a_{\max }$ is the upper bound of the gene $a_{i j} ; a_{\min }$ gene $a_{i j}$ the lower bound; $r_{2}$ is a random number; $g$ is the current iteration number; $G_{\max }$ is the maximum number of evolution; $r$ is a random number $[0,1]$ between. If the error is specified accuracies, the end, otherwise, go to step 3 .

\section{YANGTZE RIVER RO-RO TRANSPORTATION MARKET VOLUME FORECAST BASED ON GABP}

\subsection{Sample Selection}

Through the years 2001-2013 Yangtze River Ro transportation market statistics to test the reliability of genetic BP network model. 
First, select the type of information you need to enter the input: the number of ships for X1 (the number), a single ship carrying capacity of X2 (spaces), with a total capacity of X3 (spaces), and total annual revenues of X4 (ten thousand yuan); and secondly to determine the output type layer paper selects the most representative annual volume ( $Z$ cars). Finally, a method to determine the corresponding input and output of information, due to historical data are very similar to each other, so you can use the number of ships that year, four different types of information data sheets ship carrying capacity, total capacity, the total income to predict the next in the annual volume.

Firstly, choose the number of ships 2001-2010, a single ship carrying capacity, total capacity, the total income data as input, 2002-2011 annual volume as output information, and use it as a sample for training and learning, then using the previous ten years set of data to predict 2012's annual volume. The original data lists in Table 1.

\subsection{Determine BP Neural Network Topology}

Determined from Fig. (1), the input layer node is 4 and the output layer nodes is 1 in BP neural network, and according to the formula 1 , the hidden layer nodes $n=4, m=1$ into the formula 1 can be calculated the results of between 7 and 8 , according to the hidden layer nodes experience to 7 . So the whole neural network topology is the 4-7-1, a total of $4 \times 6+6 \times 1=30$ neural network weights, $6+1=7$ threshold, so the genetic algorithm code length is $30+7=37$ individuals.

\subsection{GA Optimize the Initial Weights and Thresholds}

Set the size of the population using genetic algorithms sizepop $=100$, crossover probability pcross $=[0.3]$, the mutation probability pmutation $=[0.1]$, the number of iterations is 50. Chromosome Selection operation using roulette method is to require mandatory maximum fitness value directly involved in chromosome crossover and mutation. The initial value of the input, after 50 iterations, automatic optimized neural network weights and thresholds.

\subsection{Realization and Analysis of GABP Model}

Connection weights and thresholds generation obtained by the genetic algorithm into the BP neural network, the network parameters learning efficiency $1 \mathrm{r}=0.1$, the number of iterations epochs $=10000$, training goals goal $=0.000001$, using trainlm function to train the network, call Matlab2012b tools box [net, per2] = train (net, inputn, outputn) runs the algorithm, the program can be obtained operating results: Fig. (3) genetic BP network prediction error, Fig. (4) genetic BP network prediction error percentage and Fig. (5) the desired output of genetic BP predicted output and genetic networks BP network the desired output.

Shown in Fig. (3), it can be seen in the course of running the program, the error form a curve, increased first and then decreased after reaching apex, if the sample is large enough, then the BP network prediction error can be caused by continuing to reduce straight ignored. Shown in Fig. (4), in the course of the program is running, BP neural network prediction error percentage began to fall from the highest point of a
Table 1. Yangtze River Ro transport market statistics for 2001-2011 Annual

\begin{tabular}{|c|c|c|c|}
\hline Year & $\begin{array}{c}\text { Number of } \\
\text { Ships }\end{array}$ & $\begin{array}{c}\text { Single Boat } \\
\text { Carrying } \\
\text { Capacity/Spaces }\end{array}$ & $\begin{array}{c}\text { The Total } \\
\text { Capacity/Spaces }\end{array}$ \\
\hline \hline 2001 & 80 & 27 & 2160 \\
\hline 2002 & 69 & 47 & 3243 \\
\hline 2003 & 85 & 60 & 5100 \\
\hline 2004 & 96 & 60 & 5760 \\
\hline 2005 & 106 & 60 & 6360 \\
\hline 2006 & 106 & 60 & 6360 \\
\hline 2007 & 100 & 60 & 6000 \\
\hline 2008 & 100 & 60 & 6000 \\
\hline 2009 & 72 & 60 & 4320 \\
\hline 2010 & 72 & 60 & 4320 \\
\hline 2011 & 72 & 60 & 4320 \\
\hline
\end{tabular}

(1-2)

\begin{tabular}{|c|c|c|c|}
\hline Year & $\begin{array}{c}\text { Total } \\
\text { Income/ } \\
\text { Million }\end{array}$ & $\begin{array}{c}\text { Annual Freight } \\
\text { Volume/Vehicle }\end{array}$ & $\begin{array}{c}\text { The Total } \\
\text { Transport } \\
\text { Capacity/Vehicle }\end{array}$ \\
\hline \hline 2001 & 20000 & 93900 & 46629 \\
\hline 2002 & 24000 & 145300 & 91400 \\
\hline 2003 & 48700 & 234800 & 197520 \\
\hline 2004 & 64000 & 314400 & 230700 \\
\hline 2005 & 69200 & 309185 & 276900 \\
\hline 2006 & 86700 & 399424 & 431920 \\
\hline 2007 & 100540 & 459392 & 431920 \\
\hline 2008 & 114380 & 519360 & 431920 \\
\hline 2009 & 108220 & 479328 & 431920 \\
\hline 2010 & 122060 & 439296 & 431920 \\
\hline 2011 & 135900 & 409264 & 431920 \\
\hline
\end{tabular}

positive number, down to the lowest point of negative upward trend, overall percentage error close to zero in a smallscale fluctuations, and the data are relatively close, hovering near zero, indicating that there are both positive and negative error, same token predicted large value smaller than the expected time, due to the BP network prediction error percentage of the overall genetic approaches zero, indicating that the neural network to predict the overall error in controllable range. 


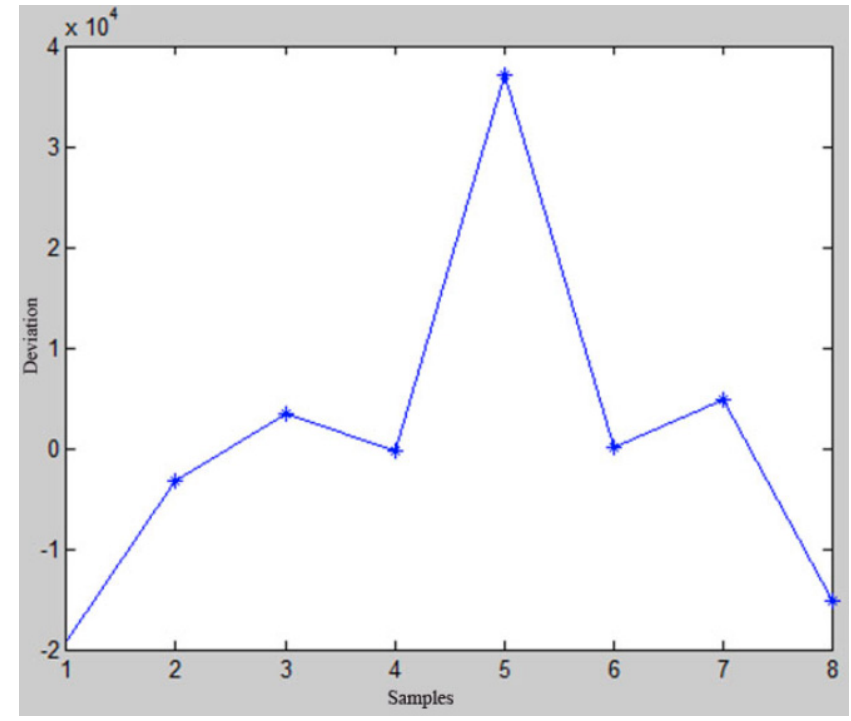

Fig. (3). BP network prediction error.

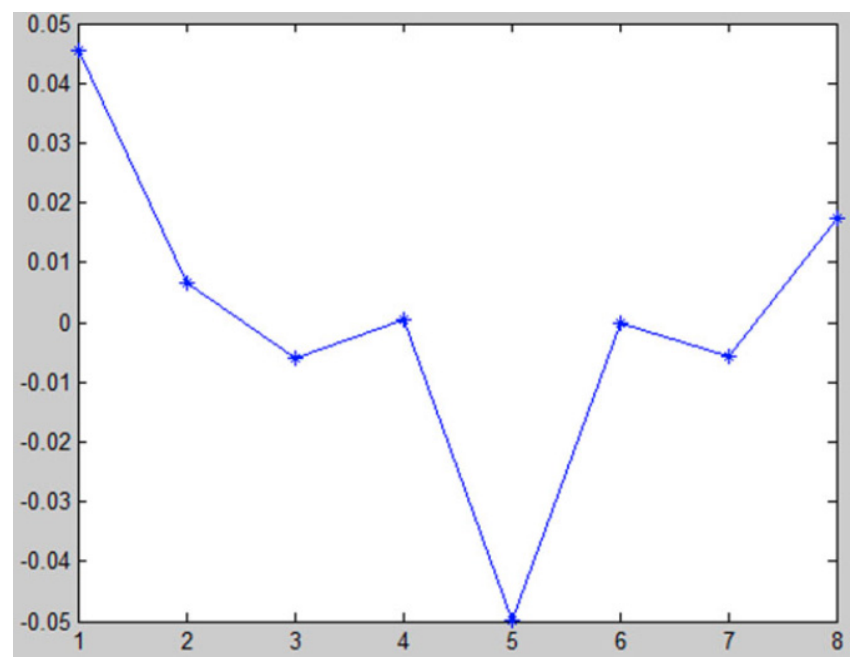

Fig. (4). Percentage of neural network forecasting error.

As can be seen from Fig. (5) Genetic BP network prediction output curve and genetic BP network desired output curve tends to overlap, although only individual point values do not coincide but little difference in the overall look of the sample predicted output and desired output basic agreement, Thus genetic BP network model has a certain accuracy and precision.

The first graph and the fourth graph of F Fig. (6) shows: GA-BP network training curve substantially through the actual data, on behalf of training and actual results roughly equal, indicating that the BP neural network model of learning and training process has validity, training convincing results, the model can be seen more in line with the actual situation, to reflect changes in the law of the Yangtze River Ro-Ro cargo transportation market.

The results show that the data for the program to run 678,030 (vehicle), indicating that the Yangtze River in 2012 predicted Ro-Ro cargo truck transport market for 429,704 (vehicles), converted cargo to $3.46 * 10^{4}$ t. The investigation shows that in 2012 the actual Yangtze River Ro cargo transportation market for 673,700 (units), by comparing predicted and actual data fairly. Based on the above analysis, we can see a higher accuracy genetic BP network models and we can get closer to the predictions and actual results.

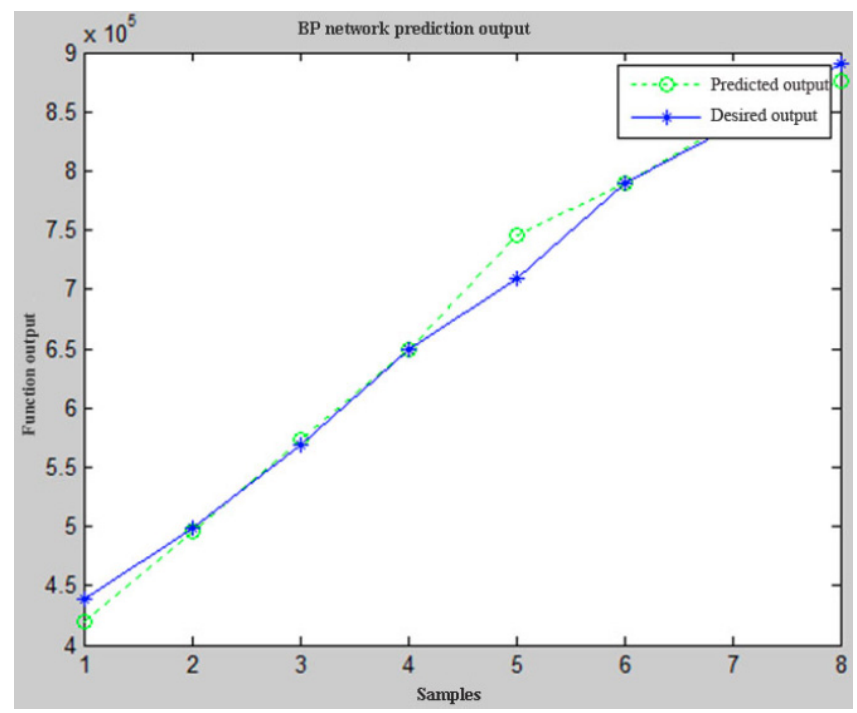

Fig. (5). Predicted output and desired output.
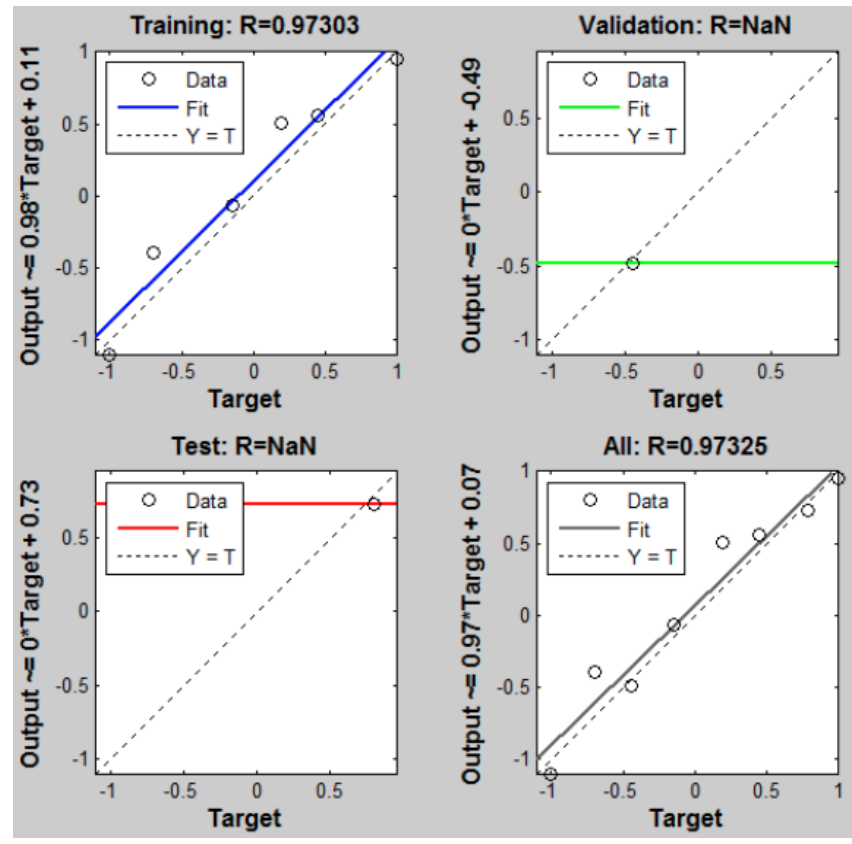

Fig. (6). Training program results show.

\section{CONCLUSION}

The BP neural network is used to predict the Yangtze River Ro-Ro cargo transportation market. On the prediction accuracy and convergence speed is higher than the predicted results from a single BP neural network algorithm obtained, and the data model and the type of network used in this paper to investigate the Yangtze River Ro-Ro transportation market is applicable. Therefore, for the rapid development of the Yangtze River Ro-Ro transportation market, in terms of prediction of genetic BP algorithm has better results, the 
prediction model to better adapt to the complex of the Yangtze River Ro-Ro transport market, can accurately find the law of development of container of the Yangtze River Ro-Ro transportation market, can largely reduce the waste of resources transport capacity, improve the market competitiveness of the Yangtze River Ro-Ro transport. Genetic BP algorithm has great significance in the excavation variation Ro-Ro transport market, timely and accurately predict market movements of cargo, optimize government policy and regulation as well as planning and other aspects of development policy.

\section{CONFLICT OF INTEREST}

The authors confirm that this article content has no conflict of interest.

\section{ACKNOWLEDGEMENTS}

This work was financially supported by Li Qiang and Zhang Wei.

\section{REFERENCES}

[1] S. Xiao, W. Wang, and B. Wang, "Study on Forecasting Methods of Highway Port Cargo Volume," ICLSIM, china,. vol. 3, no. 5, pp.831-834, 2010.

[2] G. Wang, H. Liu, and Y. Wang, "A Magnified BP Algorithm with Fast Convergence", Computer Simulation, china, vol. 25, no. 3,pp.162-172, 2008 .

[3] P. Gong, S. Ji, and W. Wei, "Based on BP Artificial Neural Network regional ecological warning," Agricultural Research, china, vol.30, no.7, pp.211-216, 2012.

[4] S. Q. Yuan, Y. N. Shen, and J. Zhang, "Based on improved BP neural network composite impeller centrifugal pump performance prediction", Agricultural Machinery, china, vol.25, pp.211-216, 2009.

Received: June 10, 2015

Revised: July 29, 2015

Accepted: August 15, 2015

(c) Jie et al.; Licensee Bentham Open

This is an open access article licensed under the terms of the Creative Commons Attribution Non-Commercial License (http://creativecommons.org/licenses/by-nc/3.0/) which permits unrestricted, non-commercial use, distribution and reproduction in any medium, provided the work is properly cited. 